\title{
EL EXPERIMENTO CHILENO EN EDUCACION: ¿Conduce a mayor equidad y calidad en la educación?
}

JESÚS M. REDONDO*

\begin{abstract}
RESUMEN
Resulta ciertamente complejo analizar simultáneamente todas las aristas de lo que ha ocurrido en la educación chilena en los últimos quince años. Existen ya estupendos antecedentes, los más recientes el informe de la OCDE (2004), de gran profundidad y finas sugerencias; y el informe Cox (2004) muy completo en antecedentes, pero quizás, en nuestra opinión, avalada por los datos de ambos informes y los resultados de nuestras investigaciones ya publicados (Redondo et al., 2004), bastante autocomplaciente con las políticas realizadas y los efectos de las mismas. Ambos informes y las investigaciones conviene leerlos simultáneamente. Aunque quizás convenga partir con el excelente artículo de Espinoza (2003): «Perspectivas alternativas en torno a la relación educación-estado, reforma educacional y política educacional: una visión crítica». La intención de este artículo no es resumir ni comentar ninguno de los informes, ni el artículo aludido; sino simplemente dar otra opinión, intentando fundamentarla en las evidencias disponibles. Es cierto que el esfuerzo realizado en la década pasada en torno a la educación en Chile es excepcionalmente significativo, más allá de los resultados. Pero queremos adentrarnos en un análisis en profundidad, o desde la profundidad (subversivo), por debajo de lo que se ve; de contenido ideológico, ético y político; con evidencias no sólo econométricas.
\end{abstract}

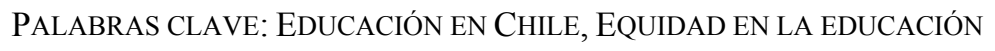

\section{A EXPERIÊNCIA CHILENA EM EDUCAÇÃO: Conduz a maior eqüidade e qualidade na educação?}

RESUMO

Certamente resulta complexo analisar simultâneamente todas las arestas do que ocorreu na educação chilena nos últimos quinze anos. Existem já excelentes antecedentes, os mais recentes; a informação da OCDE (2004), de grande profundidade e finas sugestões, e a informação do Cox (2004) muito

* Académico Universidad de Chile, Santiago, Chile. Facultad Ciencias Sociales, Departamento de Psicología. E-Mail: jredondo@uchile.cl. 
completa em antecedentes, porém, en nossa opinião, avaliada pelos dados de ambas informações e os resultados de nossas pesquisas já publicados (Redondo et al., 2004), bastante autocomplacente com as políticas realizadas e os efeitos das mesmas. Ambas informações e as pesquisas, convêm serem lidas simultâneamente. Contudo, convem partir do excelente artigo de Espinoza (2003): «Perspectivas alternativas em torno da relación educaçãoestado, reforma educacional e política educacional: uma visão crítica». A intenção deste artículo não é resumir nem comentar nenhuma das informações, nem o artigo aludido; e sim, simplemente dar outra opinião, tentando fundamentá-la nas evidencias disponíveis. Éstá claro que o esforço realizado na década passada en torno da educação no Chile foi excepcionalmente significativo, bem além dos resultados. Porém queremos entrar em uma análise mais profunda, ou partindo da profundidade (subversiva), por debaixo do que se ve; de conteúdo ideológico, ético e político; com evidencias não só estatísticas (econométricas).

PALAVRAS CHAVE: EdUCAÇÃo No CHILE, EQUIDADE NA EDUCAÇÃO

THE CHILEAN EXPERIMENTATION IN EDUCATION: Does it conduct greater equity and quality in the education?

\begin{abstract}
It is certainly complicated to simultaneously analyse all the edges in Chilean Education in the last fifteen years. There already exists great backgrounds. The most recent report of the OCDE (2004), is of great depth and fine suggestions; and the report of Cox (2004) is very complete in background, but maybe, in our opinion, vouched for by the data of both reports and the results of our investigations already published (Redondo et al., 2004), in our opinion, seem quite self-satisfied with the politics they carried out and their effects. It is convenient to read both reports simultaneously. Although, maybe it convenient to start with the excellent article of Espinoza (2003): «Alternative perspectives in relation to the educational-state, reformed education and political education: a critical vision». The intention of this article is to neither summarize nor comment on any of the above-mentioned reports; but simply to give another opinion, trying to support it with available evidences. It is certain that the effort carried out in the past decade as regards to Education in Chile is exceptionally significant, beyond the results. But we want to enter into a profound analysis, or into depth (subversive), under what is seen; of ideological content, ethic and political; with more than econometric evidence.
\end{abstract}

KEY WORDS: EDUCATION IN CHILE, EQUALITY IN EDUCATION 


\section{EL MITO DE LA IGUALDAD DE OPORTUNIDADES}

ESTE MITO ES DE larga data, y también ha sido aplastado innumerables veces por los estudios tanto cuantitativos como cualitativos en la sociología de la educación. La corriente crítica y de las teorías de la reproducción (Redondo, 2000a, 2000b) da cuenta de esto desde hace ya tres décadas. Las excepciones en individuos, algunos grupos sociales, algunas escuelas, e incluso algunos países; no contradicen la regla general, sino que la confirman como excepciones estadísticas de regularidades persistentes: los sistemas educativos no favorecen la igualdad de oportunidades (Redondo, 1997a; Dávila et al., 2005).

Con las corrientes conservadoras y neoliberales, en alza a partir de los años 70-80, surge con fuerza el planteamiento de las «escuelas eficaces», pretendiendo demostrar que existen factores que explican la posibilidad de la igualdad de oportunidades en educación, por medio de insistir en la necesidad de la calidad de las escuelas y los profesores. Los datos presentados por la corriente de las escuelas eficaces (Reynolds et al., 1997; Redondo, 1997a) parecen confirmar esta posibilidad, también señalada por el Informe de capital humano de Chile (Brunner y Elacqua, 2003). Pero estos datos y variables se refieren a escuelas específicas de países específicos, con culturas escolares y tradiciones particulares, con organizaciones sociopolíticas y estructuras de distribución de ingresos peculiares; en general, países desarrollados. ${ }^{1}$

Por otra parte, conocer las características de las escuelas que tienen buenos resultados de aprendizaje, no es un gran aporte, ya que el problema de la política educativa, y por tanto de la pretendida «igualdad de oportunidades», consiste en cómo mejorar las escuelas (Reynolds et al., 1997); cómo hacer procesos de cambio en esas instituciones que por sí mismas no tienen instaladas esas características que se reconocen en las escuelas eficaces.

Intentar trasladar las características propias de las escuelas eficaces a otras que no lo son, además de una pretensión inútil, puede resultar ineficiente e imposible (Fullan, 2002 y 2004). Estas características psicoculturales (López, 2000) no son «transportables», más bien forman parte de «identidades colectivas» construidas en largos proce-

1 Acaba de aparecer el libro: ¿Quién dijo que no se puede? Escuelas efectivas en sectores de pobreza. UNICEF/MINEDUC, 2004, que señala que también en Chile el 1\% de las escuelas vulnerables son «escuelas eficaces». 
sos psicosociales y sociopolíticos. Pretender un uso «instrumental» de las mismas, sin asumir su complejidad, y sobre todo su proceso de construcción social desde la libertad y sentido de las subjetividades de los actores sociales, es además de imposible, una aberración ética y antropológica.

La mejora escolar siempre es un camino lento y participativo; algo que el diseño de las políticas educativas de la reforma en Chile quizás nunca consideró suficientemente. Como tampoco consideró adecuadamente, ${ }^{2}$ las características, historia y procesos psicosociales de la profesión de profesor en Chile, ni las competencias de los profesores concretos y reales disponibles (OCDE, 2004:115ss). No tuvo suficientemente en cuenta la cultura autoritaria instalada en las escuelas. O la tuvo parcialmente en cuenta al plantear el cambio necesario para la interacción y reconocimiento de las culturas juveniles en los liceos; pero no para cambiar los modelos de gestión escolar de los directores administradores de los centros públicos; o de las incapacidades de gestión de la mayoría de las municipalidades. Es fácil «tirar balones fuera», pero la responsabilidad de un diseño de reforma educativa debía haber contemplado estos elementos de forma sustantiva desde el comienzo; ya que las políticas públicas consisten tanto en lo que se hace como en «lo que no se hace». Tampoco se consideró a los padres y madres, o sólo en la medida en que se les consideró sujetos de mercado (inversores o consumidores de educación), pero no tanto como ciudadanos con derecho a la educación y a la participación. Es decir, los sujetos sociales no estuvieron presentes, o muy poco o inadecuadamente presentes en la política educativa. Ésta más bien fue articulada de forma pragmática, tecnocrática, «modernizante», internacional; pero bajo la mirada y presión de los grupos de élite que dejaron hacer, consensuaron según sus intereses, y retocaron las orientaciones de la política a través de los medios de comunicación y la «intelectualidad educacional orgánica» de las fundaciones privadas de la derecha, controlando cualquier desviación.

La realidad es que las escuelas eficaces y la teoría educativa que las acompaña no son la panacea para la calidad educativa, por al menos dos motivos principales.

a) La teoría de las escuelas eficaces no está comprobada; más bien, como señala Belleï (Cox 2004:198), lo que está comprobado es

2 Quizás en los últimos años de la década de los 90 inició un mayor esfuerzo de consenso con el gremio de los profesores. 
la teoría de la reproducción: «sólo el contexto socioeconómico familiar puede considerarse como variable transnacional»; el resto de variables «referidas a las dimensiones escolares tienen una incidencia menor, sólo muestran efectividad en algunos contextos y no siempre se comportan en el mismo sentido de un país a otro (Effective Schools in Science and Mathematics, Martín et al., IEA, 2000)». Por otra parte, la investigación latinoamericana reciente (Casassus, 2003) reporta cómo son los procesos al interior de las escuelas y las aulas los que tienen más relación con la calidad de los aprendizajes, siendo éstos (todos ellos) de naturaleza psicosocial: ${ }^{3}$ especialmente el clima emocional de aula; o lo que se llama también clima escolar (Cornejo y Redondo, 2001); fundado en las expectativas de los sujetos sociales que interactúan en el espacio social de la escuela y en los procedimientos de interacción fundamentales: diseño de las tareas, evaluación y expectativas de los actores (Redondo, 1997). ${ }^{4}$

Esto añade «leña al fuego» de la crítica sobre las políticas de la reforma en educación en Chile, que parecen haber sido realizadas más mirando hacia fuera (supuesta evidencia internacional) que propiciando una investigación educacional propia (OCDE, 2004:154ss), de nuestros contextos, de la realidad de las escuelas de Chile, más allá de las estadísticas y de la «realidad virtual» de la «literatura científica de corriente principal». ${ }^{5}$

b) Las escuelas eficaces tienen que someterse al escrutinio público para responder a la pregunta: ¿eficacia para quién? (Slee, Weiner y Tomlinson, editores, 2001). Es decir, la evidencia internacional y chilena en los procesos de reforma educativa no tiene transparencia suficiente para dejar ver quién saca ventaja de la pretendida eficacia de las escuelas; o más bien lo oculta.

3 En el anexo del libro (Redondo et al., 2004), se presenta un documento realizado para intentar conceptualizar lo psicosocial dentro de la asesoría del Equipo de Psicología Educacional de la Universidad de Chile al Programa Liceo para Todos (2003).

4 Es muy ilustrativo del conflicto subyacente a las escuelas el contrastar las expectativas de los profesores, los alumnos y los padres en las encuestas de opinión a los actores educativos (CIDE, $2001 \mathrm{y} \mathrm{ss).}$

5 En el anexo del libro (Redondo et al., 2004) se presenta una conferencia sobre «Las demandas a la investigación educacional desde la globalización y la sociedad del conocimiento» en Chile, presentada en el marco del XVII Encuentro Nacional y III Internacional de Investigadores en Educación, CPEIP, 2003. 
Más años de escolaridad (cobertura, retención, doce años) parece un bien indiscutible para todos; pero las condiciones de inequidad en que ocurre la escolarización, más bien justifican el éxito escolar de los ya destinados socialmente (desde sus familias) para el éxito, y culpabilizan el fracaso escolar de los desfavorecidos; convirtiéndose en una instancia de psicologización de los problemas sociales y de legitimación de un orden social y económico inequitativo, injusto y antidemocrático (Redondo, 1997, 1998a, 1998b, 1999a, 1999b, 2000a, 2000b, 2000c, 2001c, 2002c; Dávila et al., 2005).

Más años de escolaridad (doce años), desde los estudios empíricos econométricos reportan una mejora de salarios, una tasa de retorno significativa de la inversión, etc. Pero no existe evidencia, ${ }^{6}$ que garantice que en el futuro vaya a continuar así para todos los egresados de enseñanza media, sobre todo si todos los ciudadanos son egresados de enseñanza media; más bien, en la evolución del actual modelo de acumulación capitalista globalizado, podemos prever que ocurrirá lo contrario: doce años de escolaridad será condición necesaria, pero no suficiente para mejores salarios y mayores tasas de retorno. Eso sí, la productividad que se acumulará por cada ciudadano de doce años de escolaridad será mayor; y esa acumulación la realizarán cada vez menos ciudadanos conforme a la regla universal de la acumulación capitalista del neoliberalismo, empíricamente comprobada en todo el mundo: «cada vez más ciudadanos tienen menos y cada vez menos ciudadanos tienen más».

Pero no sólo tiene que ver con los años de escolaridad, también con los contenidos y habilidades que se «educan». Es evidente, e incluso se defiende con energía, que la orientación de la reforma está enfocada en mejorar las capacidades (o competencias) productivas de los ciudadanos (incrementar su empleabilidad), para poder competir internacionalmente y aprovechar los tratados de libre comercio. Ciertamente no es un objetivo menor, pero hay que leerlo en el contexto de lo señalado más arriba. ¿Cómo legitimar más explotación? ¿O debemos agradecer que se nos quiera explotar, bajo la consideración de

6 Es evidente que no puede haber evidencia de datos del futuro. Precisamente por eso debemos desconfiar de la presunta evidencia de los datos actuales para definir el futuro de forma lineal. La ciencia «dura» en temas de futuro parece ciega; y la educación es siempre para el futuro. No es posible dejarla en manos de una ciencia ciega como la economía; es preferible contrastar el futuro con la ética, la filosofía, etc. 
que peor sería si estuviéramos excluidos de la explotación de los mercados internacionales?

Se señala también que los contenidos y habilidades deben favorecer capacidades ciudadanas que mejoren la convivencia social, que legitimen el orden social, que desarrollen la democracia, etc. Tampoco es un objetivo menor, pero no se ve mucha praxis de políticas educacionales que potencien este enfoque; más bien parece muy secundario, ya que ni siquiera se tiene en cuenta en la evaluación, ni en el SIMCE (Sistema de Medición de la Calidad de la Educación) ni en los incentivos a los profesores...; parece más bien un tema de «discurso de política educacional» y de «documentos oficiales de currículum», que un objetivo real. Pero resulta que es éste un objetivo que tendría sentido para profundizar la democracia de la sociedad chilena y poder legitimar, en el contexto socioeconómico señalado más arriba, un «proyecto país» que aglutine voluntades y conciencias. La ausencia real de este objetivo se refleja en que un 50\% de los jóvenes chilenos no considere la democracia como mejor sistema de gobierno (INJUV, 2002) o que esta democracia que tenemos en nuestro país debe mejorarse sustancialmente (INJUV, 2004); también en la poca confianza de los jóvenes, y de los ciudadanos en general, en las instituciones sociopolíticas; $\mathrm{y}$ en la poca confianza interpersonal entre los ciudadanos (PNUD, 2002; OCDE, 2004).

Por último, otro objetivo macro tiene relación con el «desarrollo personal» de los ciudadanos, tampoco evaluado de forma consistente en el proceso de reforma. Es más, la evaluación del SIMCE no incluye los objetivos transversales fundamentales obligatorios, ${ }^{7}$ y se concentra en aprendizajes instrumentales (lenguaje y matemáticas, principalmente), enfocados a las competencias productivas, más que a las competencias ciudadanas, salvo que ser ciudadano se reduzca (como señala la corriente neoliberal y postmoderna) a ser consumidores y productores; es decir, sujetos de mercado.

7 En el anexo del libro (Redondo et al., 2004) se presenta también una reflexión sobre los objetivos transversales y sobre la forma de investigación educacional con motivo de la presentación de un libro del PIIE, en el que se da cuenta de la realidad de la incorporación de los Objetivos Transversales en el área de lenguaje, en el marco del Seminario Internacional sobre «Reformas curriculares de los 90 y construcción de ciudadanía», marzo 2003. 
¿Dónde queda un enfoque integral de la educación? ¿Dónde queda el enfoque constructivista aplicado a la educación y al aprendizaje? ¿Dónde una visión actualizada y múltiple de la inteligencia y las habilidades humanas? (Gardner, 2001) ¿O una visión actual de los procesos de construcción del conocimiento en la interacción social (socioconstuctivismo) $?^{8}$

El evaluar la escolarización sólo con el SIMCE es «la muerte de la educación» y sus potencialidades para apoyar el cambio social en dirección a más igualdad («crecer con igualdad»), más ciudadanía y más humanidad; reduciendo la escolaridad a más mercado y más productividad; y por tanto, haciendo imposible la igualdad de oportunidades. Quizás por eso es tan apoyada, por determinada ideología, esta «sed evaluadora» bajo estándares. ${ }^{9}$

\section{LA FALACIA DEL MERCADO EDUCATIVO}

La política educativa de la última década larga, ha mostrado avances significativos en inversión, en mejorar los sueldos de los profesores, en mejorar las infraestructuras e insumos para la educación, en nuevos currículos, etc. Al tiempo ha aumentado la cobertura, la retención, la calidad e incluso los aprendizajes en la medida posible para las condiciones de aumento de cobertura en los sectores más desfavorecidos (Cox, 2004; OCDE, 2004; Redondo et al., 2004). Sobre esto parece no haber dudas.

8 Las más recientes investigaciones y teorías sobre aprendizaje e inteligencia humana afirman la evidencia que se produce «una construcción social del conocimiento, pero se da una apropiación individual del mismo» (Coll, 2001); que la escuela premia, y mediante esto estratifica y jerarquiza el orden social, legitimándolo. Estamos ante la tercera vuelta de tuerca: a la apropiación de la productividad del trabajo social por el capital privatizado (primera vuelta), le siguió la apropiación del interés del dinero (ahorro de los pequeños ahorradores y accionistas) por el capital financiero internacional y los bancos (segunda vuelta); y ahora la apropiación del conocimiento socialmente construido (tercera vuelta), a través de su apropiación por determinados individuos. Está por ver qué puede significar que, además, la inteligencia está socialmente distribuida; y si esto evitará la explotación total.

9 Una reflexión más amplia sobre lo que hay detrás de esta «sed evaluadora» se realizó en el Encuentro Nacional de la Sociedad Chilena de Currículo (ACHCED), 2003. 
Pero todo esto ha operado sobre una falacia no basada en evidencia empírica cuando fue formulada y convertida en ley en Chile (1982-1989): el mercado de la educación. Bajo una pretendida descentralización se ocultó una precarización de la educación pública y un traslado de la responsabilidad y culpabilidad del Estado (la política) a los establecimientos privados y a los municipios (ciudadanos). Más bien la evidencia empírica del propio experimento chileno (Carnoy, 2004; OCDE, 2004; Redondo et al., 2004) viene a probar lo contrario: el mercado no existe en educación. O mejor dicho, existe mercado de la educación en su sentido verdadero: existe negocio posible con la educación. Pero no existe en el sentido de que, el dejar que la educación funcione como mercado: elección, competencia, etc., logre ahorros «reales» de inversión y logre mejor calidad de aprendizajes «reales» de los alumnos.

El mercado educativo en Chile en la década de la transición (1990-2001) ha sido un éxito, en el sentido de que ha crecido el negocio en la educación. De 1990 a 2001 hay más de mil colegios privados más, y cincuenta colegios municipales menos. El número de alumnos del sector privado subvencionado y pagado ha crecido significativamente, y el sector municipal, incluso con mayor cobertura y más retención, ha disminuido (Mineduc, 2002). En educación básica el sector municipal tiene más alumnos que el sector privado sólo por la influencia de su mayor presencia en el sector rural, donde el negocio de la educación no tiene mucho beneficio económico; aunque empieza a operar el sector benefactor de fundaciones privadas que, con objetivos más ideológicos y culturales que mercantiles, y utilizando franquicias tributarias, están «invirtiendo» en el sector rural. En enseñanza media, el sector municipal es ya minoritario respecto al privado; incluso en la Región Metropolitana es ya sólo un tercio de los colegios y de los alumnos.

Este significativo aumento del sector privado en educación, que además opera con selección de los alumnos más capaces (aunque se defiende en el discurso público la elección de centro de los padres), no ha llevado a un aumento significativamente contundente del aprendizaje de los alumnos cuando se controla por nivel socioeconómico (Cox, 2004; OCDE, 2004), o cuando se neutraliza el input (eficiencia), como hemos fundamentado en nuestras investigaciones (Redondo et al., 2004). Excepto en la Prueba de Aptitud Académica (PAA) que «importa» para la entrada en la universidad. Es posible que los sectores de mejores ingresos no estén mayormente interesados en los resul- 
tados SIMCE, ${ }^{10}$ ya que tienen asegurado el éxito de todas formas. ${ }^{11}$ Quizás los consideran una buena entretención para que «peleen» los pobres, sin que cuestionen la estructura de segmentación e inequidad del sistema educativo y social. Es posible, en cambio, que sí estén interesados en la PAA. Si no los sectores de más élite, que tienen sus propias universidades privadas donde no se exige PAA, sí los de sectores medio alto y medio, ya que de esos resultados depende el estudiar en universidades de calidad y a bajo precio, incluso con créditos o becas. Los desfavorecidos no llegan en gran número a las universidades. La competencia real está en la PAA en un sistema universitario prácticamente privatizado (Brunner, 2005), incluso en las universidades llamadas públicas, ${ }^{12}$ en lo que se refiere al mercado, ya que hay que cancelar los aranceles.

En resumen, una falacia teórica de un economista neoliberal (Milton Friedman) y la escuela de Chicago, se transformó en un experimento de política educativa en un régimen dictatorial sin legitimidad democrática; y ha operado contundentemente durante unos gobiernos democráticos que aceptaron por convicción o por omisión dicha falacia. El resultado, como ya señalamos, es de una evidencia meridiana: más mercado de la educación, más negocio en la educación.

Los padres prefieren «ser elegidos» por colegios privados, con fondos públicos, incluso si tienen que hacer algún aporte «compartido»; no tanto porque crean que esos centros ofrecen más y mejor educación (en el sentido de lo que mide el SIMCE), sino porque seleccionan las familias y los compañeros con los que interactuará su hijo (roce social). ${ }^{13}$ El problema es que creíamos estar invirtiendo como país en educación de calidad y en mejores logros de aprendizaje.

10 De hecho, los padres no lo usan como criterio importante para la elección de centro.

11 Ciertamente siempre ganan en los rankings SIMCE del país. No les va tan bien en las comparaciones internacionales donde muestran su baja calidad e ineficiencia (Belleï, 2004).

12 En cierta forma el aporte fiscal a las universidades públicas es menor, y viene a ser «como una compensación» o equiparación por el dinero fiscal que se deriva a las universidades privadas por efecto de las donaciones y del negocio inmobiliario de las mismas y sus donantes.

13 Las últimas investigaciones sobre el tipo de empleo de los egresados universitarios, dependiendo de los colegios donde estudiaron, los apellidos y todo eso..., van en la misma dirección de lo que señalamos. 
Los colegios descubrieron que para lograr mejor imagen social y poder subir el precio de su «servicio educativo», el camino más corto era hacer una buena selección de alumnos y expulsar a los «molestosos» y a los poco capaces, así el resultado SIMCE estaba asegurado.

Los profesores se percataron que su trabajo no consistía en ser «maestros», sino instructores para preparar pruebas estandarizadas, siguiendo el viejo dicho que «la educación es el examen», lo cual se reforzó en la medida en que los incentivos económicos y el reconocimiento social dependían de esa misma prueba SIMCE (el ícono de la reforma).

Los alumnos más «vivos» se dieron cuenta que podían disgustar a sus padres y a sus profesores si en «esos días» SIMCE lo hacían mal. Total, no tenía ninguna consecuencia para ellos. Ellos eran sólo «instrumentos» sin derechos, ya que la gran «ley del día anterior» (LOCE) impedía la información individual.

El negocio rueda y funciona: ¡mil colegios privados más en una década! Ahora, lo de la calidad educativa y la mejora de los aprendizajes es otro negocio, que no está claro que alguien esté interesado en él. Total en este negocio nadie gana. Los colegios pagados o compartidos quedan mal parados, son ineficientes, cobran más de lo que dan (¡medido por el SIMCE!). ${ }^{14}$

\section{3. ¿ES UN FRAUDE LA POLÍTICA EDUCATIVA?}

Después de lo señalado en los puntos anteriores queda hacerse la pregunta que encabeza este epígrafe: ¿estamos en presencia de un gran fraude? Un fraude consiste en sustraer plata sin que se den cuenta, en este caso del presupuesto de la nación. También significa dar una cosa por otra («gato por liebre»). La inversión en educación se ha triplicado en el período (Cox, 2004:45) llegando a más de 3.000 millones de dólares de los Estados Unidos (MD). De ellos el 63\% se dedica a subvenciones: $1.890 \mathrm{MD}$; que si le añadimos la inversión en políticas universales (10\% del presupuesto), podría llegar a más de $2.200 \mathrm{MD}$ para educación obligatoria (básica y media). Si tenemos en cuenta que

14 Es posible, como señalamos, que los padres no busquen lo que mide el SIMCE cuando pagan el precio de la educación. Creo que los padres lo tenemos muy claro. El error lo cometen los «policy maker» cuando pretenden medir la calidad del aprendizaje con el SIMCE. ¿O también lo tienen claro y tratan de equivocarnos, para despistarnos de lo importante? 
el mayor número de alumnos ha ido progresivamente desplazándose del sector municipal al sector privado en más de un $5 \%$ en un contexto de retención y aumento de cobertura (OCDE 2004:15), llegando casi a un $50 \%$ para cada sector (siendo en enseñanza media casi dos terceras partes privado), podríamos concluir que actualmente al menos unos 1.200 MD se transfieren al sector privado por vía de las subvenciones. $\mathrm{Si}$ añadimos un $10 \%$ por la inversión en programas universales del sector público (infraestructuras, compras de materiales educativos: textos, computadores, etc.) que son transferencias al sector privado que facilita estos insumos (hace negocio), son otros $100 \mathrm{MD}$.

En resumen, casi dos terceras partes $(1,7 / 1,0)$ del presupuesto disponible en educación básica y media operan en el sector privado, donde el negocio (máximo beneficio) está ciertamente por encima de los objetivos educacionales, como calidad o equidad. La educación se ha consolidado como un buen campo de negocio, en el que se mueve mucha plata, incluso para investigaciones de encargo y asesorías. No sabemos si es más importante en la educación chilena el «campo» de negocio o la «arena» política.

El número de colegios privados, como ya señalamos, ha aumentado en más de mil en el período. Muchos se han construido o ampliado con dineros fiscales: tanto si ha sido por subvención directa del ministerio, como con préstamos a bajo interés, o con donaciones de fundaciones que dejan de pagar impuestos. El resultado es que se desarrolla una infraestructura por todo el país de carácter privado y con orientación de mercado de la educación. Ni siquiera se sigue la política de las «concesiones», ${ }^{15}$ en las que los inversores primero invierten su dinero y luego amortizan con el negocio de la concesión; dejando la propiedad, al final, en manos del Estado.

Evidentemente no vamos a profundizar sobre este mismo tema en la educación superior (centros de formación técnica, institutos profesionales y universidades), donde lo señalado es explícito y muy conocido. Solamente destacar una frase de Carnoy: «para el 2020 Chile debería tener el $35 \%$ de la cohorte de edad graduado de la universidad. ¿Será esto posible si todas esas familias tienen que pagar por la educación de sus hijos?» (Cox, 2004:123). ${ }^{16}$

15 Es importante darse cuenta del cambio que se ha operado de aquel dicho de «gobernar es educar», al dicho de ahora: «gobernar es concesionar».

16 La última encuesta INJUV 2003 reporta sólo un $18 \%$ de los jóvenes pertenecientes al rango socioeconómico $\mathrm{ABC} 1, \mathrm{C} 2$; y otro $26 \%$ del $\mathrm{C} 3$. 
Se ha puesto en marcha un experimento «único» que, asumido por los gobiernos de la concertación, parece empezar a plantear problemas serios en equidad (segmentación) y calidad (aprendizajes); pero también en ampliación del mercado de la educación. ¿Cuánto tiene que subir el valor de la subvención para que los colegios privados acepten alumnos con menos capacidades y más necesidades educativas? ¿Será éste el motivo de fondo para estar colocando en la «agenda pública» el tema de la subvención diferenciada?

Financiar más a los niños más pobres es una opción loable, necesaria para compensar, en alguna medida, las deficiencias de input educacional y posibilitar una mejor calidad de aprendizajes. El problema es el modo. Si se hace por alumno (enfoque de vouchers), entonces los colegios particulares estarán incentivados a conseguir alumnos desfavorecidos (quintil 1 y 2), pero sólo si tienen capacidades que les permitan ser «elegidos». La consecuencia es una profundización de la segmentación, no sólo por nivel socioeconómico o familiar, sino también por capacidades de los alumnos. Dado el gusto de los padres por ser elegidos por los colegios privados, entonces no habrá ninguna resistencia y será un modo de ascenso social individual «seguro». Al mismo tiempo se reducirá la heterogeneidad de capacidades en los centros municipales que, además de socioeconómicamente desfavorecidos, se llenarán de alumnos con menores capacidades y más necesidades educativas. Una hipotética evaluación de los resultados revelará, sin duda, un aumento de eficacia y de eficiencia en los privados, incluso controlando por nivel socioeconómico y otras variables de input o contexto social. Incluso una hipotética evaluación de «valor agregado», no cabe duda que, por «poco buena» que sea la calidad de los colegios privados, reportará que estos niños aprenden más en ellos que sus compañeros en los municipales.

Claro que siempre podemos plantearnos controlar el «acceso» de los alumnos a los centros de forma aleatoria, sin permitir la selección de alumnos por los centros financiados por fondos públicos (aunque sí permitir la elección de los padres de los centros a los que quieren llevar sus hijos); tal como se hace en Europa y varios estados de EE.UU. Con esto se acabaría, progresivamente, con la segmentación social de la educación. Pero creemos que esto puede ser la mayor «herejía» que puede ser dicha en la educación chilena: lo menos es anticonstitucional. En realidad puede ser una de las únicas medidas que permitiría una verdadera «competencia» educacional; incluso un verdadero «mercado» de la calidad educativa. Pero esto no es el negocio, ni la 
competencia, ni el mercado que se pretende. Ciertamente parece un fraude en todos los sentidos. Evidentemente, nuestra intención no es crear conflictos innecesarios; menos minusvalorar el trabajo que realizan a diario los profesores en todas las aulas y colegios de Chile, ni tampoco el de los técnicos del Ministerio de la Educación o el de los políticos; nuestra única intención es "provocar» la reflexión y el debate. Ello indicará que todavía es posible un debate real sobre la educación real de Chile, sin estar pauteados por los medios de comunicación, ni los grupos de presión. Es una invitación al debate, sin miedo al conflicto, para mejorar la educación y las posibilidades de los niños más desfavorecidos de Chile y en defensa de una educación pública de calidad, porque ciertamente «la educación no es una mercancía» ( $L e$ Monde Diplomatique, 2003) y las escuelas no son empresas (Laval, 2004).

SANTIAGO (CHILE), JUNIO 2005

RECIBIDO: JUNIO 2005

ACEPTADO: JULIO 2005

\section{REFERENCIAS BIBLIOGRÁFICAS}

BELLEÏ, C. (2004): «¿Ha tenido impacto la reforma educativa chilena?». En C. Cox: Políticas educacionales en el cambio de siglo. La reforma del sistema escolar de Chile. Santiago: Editorial Universitaria.

BRUNNER, J. J. (2005): La educación superior en Chile: orientando al mercado. Santiago: Universidad Adolfo Ibáñez.

- y G. ElACQUA (2003): Informe de capital humano de Chile. Santiago: Universidad Adolfo Ibáñez.

CARnoy, M. (2004): «Las políticas educacionales de Chile desde una perspectiva internacional». En C. Cox: Políticas educacionales en el cambio de siglo. La reforma del sistema escolar de Chile. Santiago: Editorial Universitaria.

CAsassus, J. (2003): La escuela y la (des)igualdad. Santiago: Lom.

Coll, C. (2000): «Psicología, educación y psicología de la educación». En C. Coll, J. PAlacios y A. MARChesI: Desarrollo psicológico y educación. Tomo 2: Psicología en contextos escolares. Madrid: Alianza Psicología.

CORNEJO, R. y J. M. REDONDO (2001): «El clima escolar percibido por los alumnos de enseñanza media. Una investigación en algunos liceos de la región metropolitana». Última Década $\mathrm{N}^{\mathrm{o}} 15$. Viña del Mar: Ediciones CIDPA.

Cox, C.: «Las políticas educacionales de Chile en las últimas dos décadas del siglo XX». En C. Cox: Politicas educacionales en el cambio de siglo. La reforma del sistema escolar de Chile. Santiago: Editorial Universitaria.

DÁvila, O.; F. Ghiardo y C. Medrano (2005): Los desheredados. Trayectoria de vida y nuevas condiciones juveniles. Valparaíso: Ediciones CIDPA. 
EsPINOZA, O. (2003): «Perspectivas alternativas en torno a la relación Estadoeducación, reforma educacional y política educacional: una visión crítica». En A. ARrau (editor): Bases para la competencia en Chile. La educación en una sociedad desigual. Santiago: Universidad de Chile, FAcso, Programa Predes.

Fullan, M. (2004): Las fuerzas del cambio. La continuación. Madrid: Akal.

- (2002): Las fuerzas del cambio. Explorando las profundidades de la reforma educativa. Madrid: Akal.

GARDNER, H. (2001): La inteligencia reformulada. Barcelona: Paidós.

InJUV (2004): IV Encuesta Nacional de Juventud. Santiago: InJUV.

- (2002): III Encuesta Nacional de Juventud. Santiago: InJuV.

Laval, Ch. (2004): La escuela no es una empresa. Barcelona: Paidós.

Le Monde DiplomatiQue (2003): La educación no es una mercancía. Santiago: Editorial Aún Creemos en los Sueños.

López Herrerías, J. A. (2000): Paradigmas y métodos pedagógicos para la educación social. Valencia: Editorial Nau Llibres.

Mineduc (2002): Estadisticas educacionales 2001. Santiago: MineduC.

OCDE (2004): Revisión de políticas nacionales en educación: Chile. París y Santiago: OCDE Y MINEDUC.

PNud (2002): Desarrollo humano en Chile. Nosotros los chilenos: un desafio cultural. Santiago: PNUD.

REDONDO J. M. (2002): «La salud laboral docente en el contexto de los requerimientos actuales a la educación». Revista Docencia, Año 7, Nº18. Santiago: Colegio de Profesores de Chile AG.

- (2000a): «La condición juvenil entre la educación y el empleo». Última Década $\mathrm{N}^{\circ} 12$. Viña del Mar: Ediciones CIDPA.

_ (2000b): «El fracaso escolar y las funciones estructurales de la escuela: una perspectiva crítica». Revista de Sociología N ${ }^{\circ} 14$. Santiago: Universidad de Chile, FACSO, Departamento de Sociología.

_ (1999a): «La influencia de la escolarización en enseñanza media en la subjetividad de los jóvenes que fracasan en ella». Revista de Psicología, Vol. VIII, Nº1. Santiago: Universidad de Chile, FACso, Departamento de Psicología.

— (1999b): «La modernización de la educación y la psicologización de los problemas sociales: o cómo se enseña a las víctimas a culpabilizarse». Revista de Psicología, Vol. VIII, No2. Santiago: Universidad de Chile, FACsO, Departamento de Psicología.

_ (1998): «Características psicosocioeducativas de los alumnos que fracasan en primer ciclo de la enseñanza media del País Vasco». Revista Enfoques Educacionales, Vol. I., No2. Santiago: Universidad de Chile, FACsO, Departamento de Educación.

_ (1997): «La dinámica escolar: de la diferencia a la desigualdad». Revista de Psicología, Vol. VI. Santiago: Universidad de Chile, FACSO, Departamento de Psicología.

— enseñanza media. La necesidad y la posibilidad de construir comunidades educativas autónomas y responsables». Revista de Psicología, Vol. VII. Santiago: Universidad de Chile, FACSO, Departamento de Psicología.

— y C. DesCOUVIERES (2001): «De la eficacia a la eficiencia de las escuelas básicas de Chile (1990-1997). Aproximaciones a un estudio de valor agregado». Revista 
Enfoques Educacionales, Vol. III., Nº1. Santiago: Universidad de Chile, FACsO, Departamento de Educación.

— - y K. RoJAS (2004): «Equidad y calidad de la educación en Chile. Reflexiones e investigaciones de eficiencia de la educación obligatoria (19902001)». Santiago: Universidad de Chile, Vicerrectoría de Investigación, Departamento de Psicología.

— y Equipo de Psicología Educacional Universidad de Chile (2000): «A propósito de una intervención en psicología educacional». Publicación de las «I Jornadas Internacionales de Psicología Educacional: la psicología educacional en tiempos de reforma». La Serena: Universidad de La Serena.

Reynolds, D.; R. Bollen, B. Creemers, D. Hopkins, L. Stoll y N. Lagerweij (1997): Las escuelas eficaces. Claves para mejorar la enseñanza. Madrid: Santillana.

SleE, R.; G. WeINER y S. TOMLINSON (editores) (2001): ¿Eficacia para quién? Crítica de los movimientos de las escuelas eficaces y de la mejora escolar. Madrid: Akal. 